\title{
TCEC15: the 15th Top Chess Engine Championship
}

Article

Accepted Version

The TCEC15 official report

Haworth, G. and Hernandez, N. (2019) TCEC15: the 15th Top Chess Engine Championship. ICGA Journal, 41 (3). pp. 153163. ISSN 1389-6911 doi: https://doi.org/10.3233/ICG-190115 Available at https://centaur.reading.ac.uk/83156/

It is advisable to refer to the publisher's version if you intend to cite from the work. See Guidance on citing.

Published version at: https://content.iospress.com/articles/icga-journal/icg190115

To link to this article DOI: http://dx.doi.org/10.3233/ICG-190115

Publisher: The International Computer Games Association

All outputs in CentAUR are protected by Intellectual Property Rights law, including copyright law. Copyright and IPR is retained by the creators or other copyright holders. Terms and conditions for use of this material are defined in the End User Agreement.

\section{www.reading.ac.uk/centaur}

\section{CentAUR}

Central Archive at the University of Reading

Reading's research outputs online 


\section{TCEC15: the $15^{\text {th }}$ Top Chess Engine Championship}

Guy Haworth ${ }^{1}$ and Nelson Hernandez

Reading, UK and Maryland, USA

TCEC Season 15 started on March $5^{\text {th }} 2019$ with a more liberal Division 4 featuring several engines in their first TCEC season. At the top end, interest would centre on whether the recent entries, ETHEREAL, KOMODO MCTS and LEELA CHESS ZERO would again improve their already impressive performances. Fig. 1 and Table 1 provide the logos and details on the enlarged field of 44 engines.

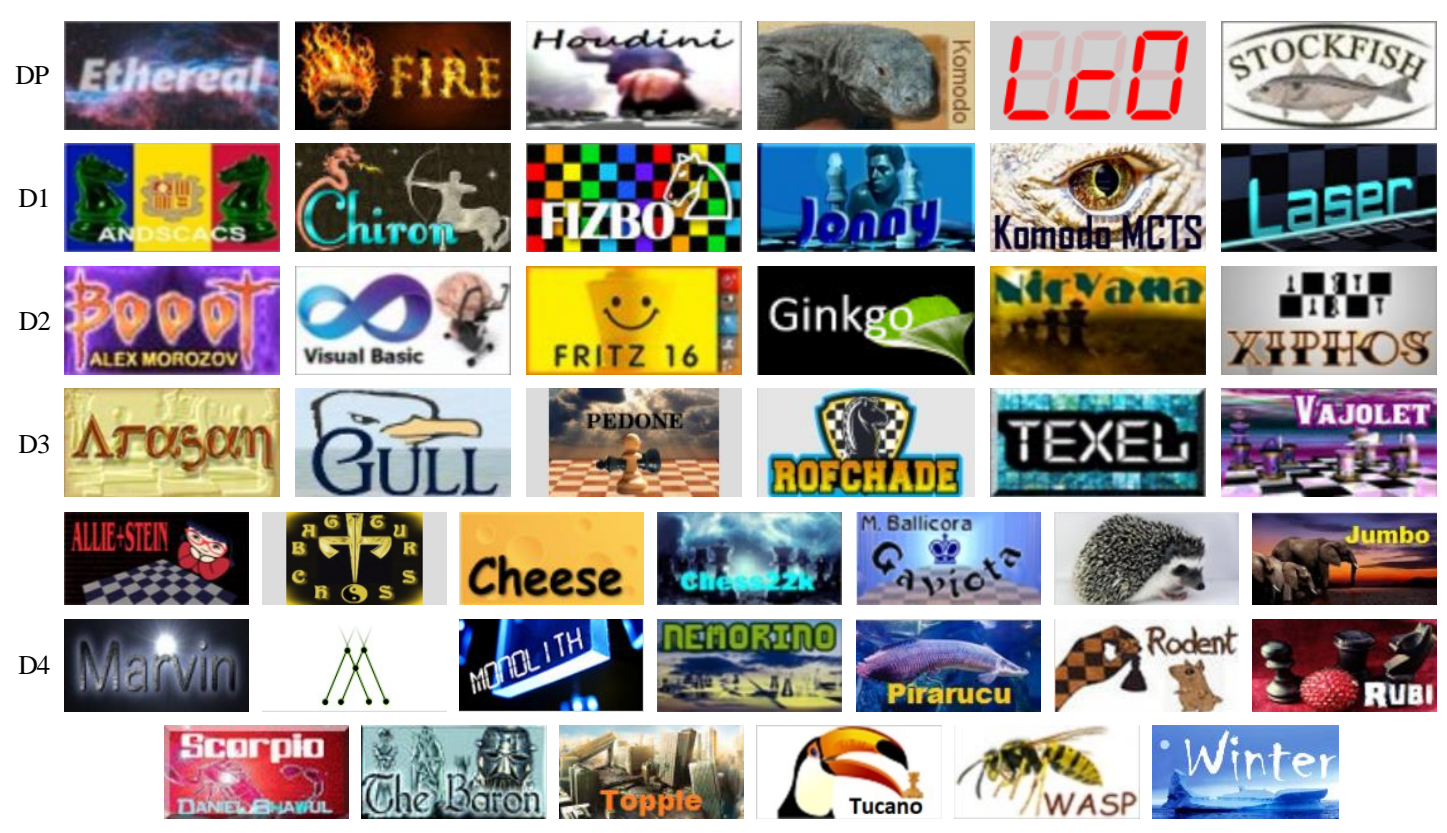

Fig. 1. Logos for the TCEC 15 engines (CPW, 2019) as in their original divisions.

There were a few nudges to the rules. In the event of network breaks, if both engines were in the 7-man and/or TCEC win (or draw) zone, the game was adjudicated as a win (or draw). Otherwise, TCEC resumed games with extra initialisation time rather than restart them.

The common platform for TCEC15, as for TCEC14, consisted of two computers. One was the established, formidable 44-core server of TCEC11-14 (Intel, 2017) with 64GiB of DDR4 ECC RAM and a Crucial CT250M500 240 GB SSD for the EGTs. The 'GPU server', upgraded to a Quad Core i5 3570k with 32GiB DDR3 RAM, sported Nvidia (2018) GeForce RTX 2080 Ti and 2080 GPUs.

\footnotetext{
${ }^{1}$ Corresponding author: g.haworth@ reading.ac.uk
} 
Table 1. The TCEC15 engines (CPW, 2019), details, authors and progress.

\begin{tabular}{|c|c|c|c|c|c|c|c|c|c|c|c|c|}
\hline \multirow{2}{*}{ \# } & \multicolumn{2}{|r|}{ Engine } & \multicolumn{3}{|l|}{ Initial } & \multirow{2}{*}{ thr. } & \multirow{2}{*}{$\begin{array}{c}\text { proto- } \\
\text { col }\end{array}$} & \multirow{2}{*}{$\begin{array}{c}\text { Hash } \\
\text { Kb }\end{array}$} & \multirow{2}{*}{ EGTs } & \multirow{2}{*}{ Authors } & \multirow{2}{*}{$\begin{array}{r}\text { Countr } \\
\text { y Codes }\end{array}$} & \multirow{2}{*}{$\begin{array}{c}\text { Final } \\
\text { Div. }\end{array}$} \\
\hline & ab & Name & Version & ELO & Div. & & & & & & & \\
\hline 01 & $\mathrm{AS}$ & AllieStein & v0.1-n4 & 2557 & $4 \mathrm{~b}$ & 3 & uci & 7,168 & - & Adam Treat and Mark Jordan & US & フォフスP \\
\hline 02 & An & Andscacs & 0.95123 & 3469 & 1 & 43 & uci & 8,192 & - & Daniel José Queraltó & $\mathrm{AD}$ & $\rightarrow 1$ \\
\hline 03 & $\mathrm{Ar}$ & Arasan & TCEC15 & 3290 & 3 & 43 & uci & 16,384 & Syz. & Jon Dart & US & $\rightarrow 3$ \\
\hline 04 & $\mathrm{Ba}$ & Bagatur & $1.5 \mathrm{f}$ & 2496 & $4 \mathrm{~b}$ & 43 & uci & $?$ & - & Krasimir Topchiyski & BG & $\searrow 5$ \\
\hline 05 & Bo & Booot & 6.3 .1 & 3407 & 2 & 32 & uci & 16,384 & - & Alex Morozov & UA & $\rightarrow 2$ \\
\hline 06 & $\mathrm{Ch}$ & Cheese & 2.0 & 2740 & $4 a$ & 8 & uci & 16,384 & - & Patrice Duhamel & FR & $\rightarrow 4$ \\
\hline 07 & $\mathrm{Cb}$ & ChessbrainVB & 3.72 & 3342 & 2 & 43 & xboard & 1,200 & - & Roger Zuehlsdorf & $\mathrm{DE}$ & $\rightarrow 2$ \\
\hline 08 & $\mathrm{c} 22$ & chess $22 \mathrm{k}$ & 1.12 & 2970 & $4 b$ & 43 & uci & 16,384 & - & Sander Maassen vd Brink & NL & $\rightarrow 4$ \\
\hline 09 & $\mathrm{Ch}$ & Chiron & 230119 & 3411 & 1 & 43 & uci & 16,384 & Syz. & Ubaldo Andrea Farina & IT & $\searrow 2$ \\
\hline 10 & Et & Ethereal & 11.38 & 3483 & $\mathrm{P}$ & 43 & uci & 16,384 & Syz. & Andrew Grant & US & $\searrow 1$ \\
\hline 11 & $\mathrm{Fi}$ & Fire & 11819 & 3488 & $\mathrm{P}$ & 43 & uci & 16,384 & Syz. & Norman Schmidt & US & $\searrow 1$ \\
\hline 12 & $\mathrm{Fz}$ & Fizbo & 2 & 3419 & 1 & 43 & uci & 16,384 & Syz. & Youri Matiounine & US & $\rightarrow 1$ \\
\hline 13 & Fr & Fritz & 16.10 & 3319 & 2 & 43 & uci & 16,384 & Nal? & Vasik Rajlich & CZ/US & $\searrow 3$ \\
\hline 14 & $\mathrm{Ga}$ & Gaviota & 1.01 & 2653 & $4 \mathrm{~b}$ & 43 & uci & 16,384 & Gav. & FM Miguel Ballicora & ES & $\rightarrow 4$ \\
\hline 15 & $\mathrm{Gi}$ & Ginkgo & 2.18 & 3440 & 2 & 43 & uci & 16,384 & Syz. & Frank Schneider & $\mathrm{DE}$ & $\rightarrow 2$ \\
\hline 16 & $\mathrm{Gu}$ & Gull & 3 & 3300 & 3 & 43 & uci & 16,384 & - & Vadim Demichev & RU & $\rightarrow 3$ \\
\hline 17 & Ho & Houdini & 6.03 & 3571 & $\mathrm{P}$ & 43 & uci & 16,384 & Syz. & Robert Houdart & $\mathrm{BE}$ & $\rightarrow \mathrm{P}$ \\
\hline 18 & Ig & Igel & 1.2 & 2412 & $4 \mathrm{a}$ & 43 & uci & 16,384 & - & Volodymyr Shcherbyna & UA & $\searrow 5$ \\
\hline 19 & Jo & Jonny & 8.1 & 3400 & 1 & 43 & uci & 16,384 & Syz. & Johannes Zwanzger & $\mathrm{DE}$ & $\searrow 2$ \\
\hline 20 & $\mathrm{Ju}$ & Jumbo & 0.6 .99 .2 & 2738 & $4 \mathrm{~b}$ & 43 & xboard & 16,384 & Gav. & Sven Schüle & $\mathrm{DE}$ & $\searrow 5$ \\
\hline 21 & Ko & Komodo & 2306.00 & 3486 & $\mathrm{P}$ & 43 & uci & 16,384 & Syz. & Don Dailey, Larry Kaufman, & US & $\rightarrow \mathrm{P}$ \\
\hline 22 & $\mathrm{Km}$ & Komodo MCTS & 2301.00 & 3487 & 1 & 43 & uci & 16,384 & Syz. & Mark Lefler & US & $\neg \mathrm{P}$ \\
\hline 23 & $\mathrm{La}$ & Laser & 230319 & 3444 & 1 & 43 & uci & 16,384 & Syz. & Jeffrey An, Michael An & US & $\rightarrow 1$ \\
\hline 24 & $\mathrm{Lc}$ & LCZero & v0.21.1-n41800 & 3603 & $\mathrm{P}$ & 3 & uci & - & Syz. & UCT/NN AI Community & - & $\rightarrow \mathrm{P}$ \\
\hline 25 & $\mathrm{Ma}$ & Marvin & 20190228 & 2678 & $4 \mathrm{~b}$ & 43 & uci & 16384 & Syz. & Martin Danielsson & $\mathrm{SE}$ & $\rightarrow 4$ \\
\hline 26 & $\mathrm{Mi}$ & Minic & 0.42 & 2872 & $4 a$ & 40 & xboard & 16384 & - & Vivien Clauzon & FR & $\rightarrow 4$ \\
\hline 27 & Mo & Monolith & 1 & 2617 & $4 \mathrm{~b}$ & 16 & uci & 16384 & Syz. & Jonas Mayr & AT & $\rightarrow 4$ \\
\hline 28 & $\mathrm{Ne}$ & Nemorino & 5.08 & 3247 & $4 a$ & 43 & uci & 16,384 & Syz. & Christian Günther & US & $\nearrow 4$ \\
\hline 29 & $\mathrm{Ni}$ & Nirvana & 2.4 & 3343 & 2 & 32 & uci & 16,384 & - & Thomas Kolarik & US & $\searrow 3$ \\
\hline 30 & $\mathrm{Pe}$ & Pedone & 1.9 & 3285 & 3 & 43 & uci & 16,384 & Syz. & Fabio Gobbato & IT & $\searrow 4$ \\
\hline 31 & pi & pirarucu & 2.9 .5 & 3124 & $4 \mathrm{~b}$ & 43 & uci & 4,096 & - & Raoni Campos & $\mathrm{BR}$ & $\rightarrow 4$ \\
\hline 32 & Ro & Rodent III & 0.276 & 2958 & $4 a$ & 16 & uci & 4,096 & - & Pawel Koziol & PL & $\rightarrow 4$ \\
\hline 33 & $\mathrm{rf}$ & rofchade & $2.016 \mathrm{~T}$ & 3290 & 3 & 43 & uci & 16,384 & Syz. & Ronald Friederich & NL & $\nearrow 2$ \\
\hline 34 & $\mathrm{Ru}$ & RubiChess & 1.3 .1 & 3200 & $4 a$ & 43 & uci & 16,384 & Syz. & Andreas Matthies & $\mathrm{DE}$ & $\rightarrow 4$ \\
\hline 35 & $\mathrm{Sn}$ & ScorpioNN & 2.9 .0 & 2325 & $4 \mathrm{~b}$ & - & xboard & - & - & Daniel Shawl & ET & $\rightarrow 4$ \\
\hline 36 & St & Stockfish & 19040612 & 3603 & $\mathrm{P}$ & 43 & uci & 16,384 & Syz. & Tord Romstad, Marco Costalba, & $\mathrm{NO} / \mathrm{IT} /$ & $\rightarrow \mathrm{P}$ \\
\hline 37 & $\mathrm{Te}$ & Texel & $1.08 \mathrm{a} 13$ & 3210 & 3 & 43 & uci & 16,384 & Syz. & Peter Österlund & SE & $\rightarrow 3$ \\
\hline 38 & $\mathrm{~Tb}$ & The Baron & 3.44 .1 & 2987 & $4 a$ & 42 & uci & 16,384 & Syz. & Richard Pijl & NL & $\rightarrow 4$ \\
\hline 39 & To & Topple & 0.4 .0 & 2615 & $4 a$ & 43 & uci & 16,384 & Syz. & Vincent Tang & UK & $\searrow 5$ \\
\hline 40 & $\mathrm{Tu}$ & Tucano & 7.07 & 2996 & $4 a$ & 43 & xboard & 16,384 & - & Alcides Schulz & $\mathrm{BR}$ & $\rightarrow 4$ \\
\hline 41 & $\mathrm{Va}$ & Vajolet 2 & 2.6 .1 & 3240 & 3 & 43 & uci & 16,384 & Syz. & Marco Belli & IT & $\rightarrow 3$ \\
\hline 42 & Wa & Wasp & 3.59 & 3101 & $4 b$ & 43 & uci & 8,192 & Syz. & John Stanback & US & $\rightarrow 4$ \\
\hline 43 & Wi & Winter & 0.5 & 2868 & $4 a$ & 43 & uci & 16,384 & - & FM Jonathan Rosenthal & $\mathrm{CH}$ & $\rightarrow 4$ \\
\hline 44 & $\mathrm{Xi}$ & Xiphos & 0.5 .2 & 3449 & 2 & 43 & uci & 16384 & Syz. & Milos Tatarevic & RS & $\lambda 1$ \\
\hline
\end{tabular}

Initial interest centred on a third non-Shannon engine, this time the hybrid ALLIESTEIN, a cross between Adam Treat's ALLIE and Mark Jordan's LEELENSTEIN (Chessdom, 2019) and a pupil of supervised rather than 'tabula rasa' learning. Would this combine the best of 'Shannon AB' and neural-network approaches in a case of ' $1+1=3$ ' or would it be a fall between two stools? There were several reasons for believing that ALLIESTEIN would not tarry in Division 4 for too long.

HANNIBAL was recuperating after its crashes in TCEC14 but new to TCEC were some other 'AB conventional' engines - CHEESE, BAGATUR, IGEL, JuMBO, MARVIn, MiniC, MONOLITH, RubiCHESS and TOPPLE - a generous addition to the line-up. Given the wide range of estimated ELOs, there was likely to be a higher degree of carnage in Division 4 and so it proved. The division was in fact split into two halves with a play-off for the promotion places between the top two in each half. 


\section{Division 4a/b: each half of 1 DRR, 2 RRs, 18 rounds, 90 games @ 30'+5"/m}

As for TCEC12-14, each engine played both White and Black from four-ply openings defined by the second author here. The results are as in Tables 2-3: 'P\%' is the \%-score and 'ELO \pm ' the change to the engine's nominal ELO based on its performance. Generic stats are in Tables 11 and 12. In part 4a, CHEESE's win 'against the otherwise unchallenged NEMORINO in game 11.2/52 was a clear outlier. RUBICHESS took second by virtue of its 2-0 result against WINTER.

In Act 2 game 12.5/60, SCORPIONN had a 7-man win $(d t m=22 \mathrm{~m})$ on move 80 but took 108 moves to get a 6-man EGT result. WASP disconnected against ALLIESTEIN in game 15.4/74, effectively a 1.5point swing for second place as 'crashes' are the first tie-breaker. Unusually, games 29 and 87 ended in mate, and SCORPIO exhibited its 'resigns' move in games 5, 28 and 78.

The network crashed in g32, ALLIESTEIN-CHESS22K, on move 49w with CHESS22K's evaluation at +5.3 , a clear case for a continuation rather than a restart, surely the default response. ALLIESTEIN worked through to a 6-man RB-BP 'mate in 18' win with some difficulty. It would have been interesting to see it actually achieve this as neither engine was using the 6-man endgame tables (de Man, 2018).

Table 2. The TCEC15 Division 4a cross-table: one DRR phase, 18 rounds, 90 games.

\begin{tabular}{|c|c|c|c|c|c|c|c|c|c|c|c|c|c|c|c|c|}
\hline \# & Engine & ELO & Pts & $\mathbf{P} \%$ & Elo \pm & SB & $\mathrm{Ne}$ & $\mathbf{R u}$ & Wi & Ro & $\mathbf{T b}$ & $\mathbf{T u}$ & Ch & Mi & To & Ig \\
\hline 01 & Nemorino 5.08 & 3242 & 15.0 & 83.3 & -9 & 120.00 & & 11 & $1=$ & $1=$ & 11 & $=1$ & $=0$ & 11 & 11 & 11 \\
\hline 02 & RubiChess 1.3 .1 & 3039 & 12.5 & 69.4 & 0 & 90.25 & 00 & & 11 & $==$ & $=0$ & $1=$ & 11 & 11 & 11 & $1=$ \\
\hline 03 & Winter 0.5 & 2911 & 12.5 & 69.4 & 25 & 92.75 & $0=$ & 00 & & 10 & 11 & $=1$ & $=1$ & 11 & 11 & 11 \\
\hline 04 & Rodent III 0.276 & 2980 & 11.0 & 61.1 & -3 & 82.50 & $0=$ & $==$ & 01 & & $=1$ & 01 & 10 & $=1$ & $=1$ & 11 \\
\hline 05 & The Baron 3.44.1 & 2943 & 10.5 & 58.3 & -1 & 71.25 & 00 & $=1$ & 00 & $=0$ & & $1=$ & $1=$ & 11 & 11 & $=1$ \\
\hline 06 & Tucano 7.07 & 2966 & 9.5 & 52.8 & -15 & 64.75 & $=0$ & $0=$ & $=0$ & 10 & $0=$ & & $=1$ & 10 & 11 & 11 \\
\hline 07 & Cheese 2.0 & 2827 & 8.0 & 44.4 & -1 & 62.75 & $=1$ & 00 & $=0$ & 01 & $0=$ & $=0$ & & 01 & 10 & 11 \\
\hline 08 & Minic 0.42 & 2677 & 5.0 & 27.8 & 0 & 30.25 & 00 & 00 & 00 & $=0$ & 00 & 01 & 10 & & 10 & $1=$ \\
\hline 09 & Topple 0.4 .0 & 2589 & 3.5 & 19.4 & 0 & 21.00 & 00 & 00 & 00 & $=0$ & 00 & 00 & 01 & 01 & & 10 \\
\hline 10 & Igel 1.2 & 2521 & 2.5 & 13.9 & 1 & 17.50 & 00 & $0=$ & 00 & 00 & $=0$ & 00 & 00 & $0=$ & 01 & \\
\hline
\end{tabular}

Table 3. The TCEC15 Division 4b cross-table: one DRR phase, 18 rounds, 90 games.

\begin{tabular}{|c|c|c|c|c|c|c|c|c|c|c|c|c|c|c|c|c|}
\hline$\#$ & Engine & ELO & Pts & $\mathbf{P} \%$ & Elo \pm & SB & pi & AS & Wa & Ma & c22 & Sn & Mo & $\mathbf{G a}$ & Ju & Ba \\
\hline 01 & pirarucu 2.9.5 & 3116 & 14.0 & 77.8 & -8 & 106.25 & & $=0$ & 10 & 11 & 11 & 10 & 11 & $1=$ & 11 & 11 \\
\hline 02 & AllieStein v0.1-n & 3200 & 13.5 & 75.0 & -216 & 108.50 & $=1$ & & 01 & 01 & $1=$ & 11 & $=1$ & $=1$ & $1=$ & 11 \\
\hline 03 & Wasp 3.59 & 3122 & 13.0 & 72.2 & -23 & 97.00 & 01 & 10 & & $==$ & $==$ & $=1$ & 11 & $=1$ & 11 & 11 \\
\hline 04 & Marvin 20190228 & 3000 & 11.0 & 61.1 & -96 & 79.75 & 00 & 10 & $==$ & & $==$ & $=1$ & $=1$ & $1=$ & $=1$ & 11 \\
\hline 05 & chess $22 \mathrm{k} 1.12$ & 2981 & 10.5 & 58.3 & -7 & 69.75 & 00 & $0=$ & $==$ & $==$ & & $==$ & 01 & 11 & 11 & 11 \\
\hline 06 & ScorpioNN 2.9.0 & 2276 & 9.0 & 50.0 & +360 & 62.25 & 01 & 00 & $=0$ & $=0$ & $==$ & & $=1$ & $==$ & $=1$ & 11 \\
\hline 07 & Monolith 1 & 2800 & 8.0 & 44.4 & -53 & 48.00 & 00 & $=0$ & 00 & $=0$ & 10 & $=0$ & & 11 & $1=$ & 11 \\
\hline 08 & Gaviota 1.01 & 2745 & 7.0 & 38.9 & -54 & 42.75 & $0=$ & $=0$ & $=0$ & $0=$ & 00 & $==$ & 00 & & 11 & 11 \\
\hline 09 & Jumbo 0.6 .99 .2 & 2600 & 2.5 & 13.9 & -215 & 21.50 & 00 & $0=$ & 00 & $=0$ & 00 & $=0$ & $0=$ & 00 & & $=0$ \\
\hline 10 & Bagatur 1.5f & 3000 & 1.5 & 08.3 & -711 & 3.75 & 00 & 00 & 00 & 00 & 00 & 00 & 00 & 00 & $=1$ & \\
\hline
\end{tabular}

The Division 4 play-off was marred and skewed by two PIRARUCU technical concessions. Throttled back by fiat from 43 threads to 16, this engine then underperformed and missed a likely second-place promotion. This should not obscure the fact that ALLIESTEIN showed new form to win, remarkably beating NEMORINO 4-0 and RUBICHESS 21/2-11/2. The only blot on ALLIESTEIN's escutcheon was its loss as Black to RUBICHESS in game 9.1/17: the power of two queens is not to be underestimated. 
Table 4. The TCEC15 Division 4 play-off cross-table: two DRR phases, 12 rounds, 24 games.

\begin{tabular}{llccccccccc}
\hline$\#$ & Engine & ELO & Pts & P\% & Elo \pm & SB & AS & Ne & pi & Ru \\
\hline 1 & AllieStein v0.1-n & 3054 & 9.5 & 79.2 & +179 & 47.50 & & 1111 & $1=1=1=01$ \\
2 & Nemorino 5.08 & 3244 & 6.0 & 50.0 & -12 & 25.50 & 0000 & & $=11=1=1=$ \\
3 & pirarucu 2.9.5 & 3111 & 4.5 & 38 & +7 & 25.50 & $0=0=$ & $=00=$ & & $1===$ \\
4 & RubiChess 1.3.1 & 3045 & 4.0 & 33 & +13 & 27.00 & $0=10$ & $0=0=0===$ \\
\hline
\end{tabular}

\section{Division 3: 2 DRR phases, 14 rounds, 112 games @ 30'+5"/m, 4-ply openings}

With LEELA and KOMODOMCTS now in the higher divisions, competition for promotion was bound to be more open and keenly contested. Indeed, each engine lost at least two and won at least three games. There were plenty of wins below the diagonal of the eventual $\mathrm{x}$-table, notably by PEDONE and VAJOLET at the expense of ROFCHADE, games 8/2.2 and 30/8.2.

Game 45, ALLIESTEIN-ARASAN, broke the TCEC shortest-win record in g45/12.1 with a mate in 20 moves, ${ }^{2,3}$ too sudden and savage for the TCEC referee to intervene. This was not the first evidence that there are still some bugs to be fixed. ALLIESTEIN was weak on moves 4 and 7 and blundered on 15 and 18 - the $\mathrm{m} 4 / \mathrm{m} 7$ mistakes apparently connected to it failing to recognise castling options.

Despite this, ALLIESTEIN stayed in the promotion fight and its chances were conclusively enhanced with the 0-1 result of g100/50.2, ARASAN-ROFCHADE in which ARASAN got tangled up. The final results contained some surprises. No tiebreak rules were needed and ROFCHADE deservedly took first place after being unbeaten by the top half of the table including a unique 4-0 result against TEXEL. In contrast, ALLIESTEIN in second lost its matches against ARASAN and TEXEL but was unbeaten by the bottom four engines. The hope was that its known weaknesses had been sorted out. As the underperforming GULL was the reference engine at 3300 in the TCEC ELO scale, all other engines increased their TCEC ELO ${ }^{4}$ even NEMORINO which returned to Division 4. Wool (2019) picked out the round 19 game ARASAN-ALLIESTEIN which ARASAN would have ended with position-repetition but which in fact ended in a 6-man EGT-adjudicated draw.

Table 5. The TCEC15 Division 3 cross-table: two DRR phases, 28 rounds, 112 games.

\begin{tabular}{|c|c|c|c|c|c|c|c|c|c|c|c|c|c|c|}
\hline \# & Engine & ELO & Pts & P\% & Elo \pm & SB & ro & AS & Ar & Te & Va & Gu & $\mathbf{P e}$ & $\mathrm{Ne}$ \\
\hline 1 & rofChade $2.016 \mathrm{~T}$ & 3290 & 18.0 & 64.3 & +57 & 239.75 & & $====$ & $===1$ & 1111 & $=0==$ & $==11$ & $0==1$ & $=11=$ \\
\hline 2 & AllieStein v0.2-n4 & 3233 & 16.5 & 58.9 & +95 & 219.25 & $====$ & & $=0==$ & $=001$ & $=11=$ & $1===$ & $=111$ & $1===$ \\
\hline 3 & Arasan TCEC15 & 3302 & 15.5 & 55.4 & +32 & 209.75 & $===0$ & $=1==$ & & $0=10$ & $1=01$ & $===1$ & $==1=$ & $===1$ \\
\hline 4 & Texel 1.08a13 & 3210 & 13.5 & 48.2 & +55 & 182.00 & 0000 & $=110$ & $1=01$ & & $=0==$ & $==01$ & $=101$ & $10=1$ \\
\hline 5 & Vajolet2 2.6.2 & 3240 & 13.0 & 46.4 & +35 & 184.00 & $=1==$ & $=00=$ & $0=10$ & $=1==$ & & $====$ & $1=0=$ & $=001$ \\
\hline 6 & Gull 3 & 3300 & 12.5 & 44.6 & 0 & 171.00 & $==00$ & $0===$ & $===0$ & $==10$ & $====$ & & $==1=$ & $10==$ \\
\hline 7 & Pedone 1.9 & 3285 & 12.0 & 42.9 & +3 & 165.50 & $1==0$ & $=000$ & $==0=$ & $=010$ & $0=1=$ & $==0=$ & & $==11$ \\
\hline 8 & Nemorino 5.08 & 3232 & 11.0 & 39.3 & +31 & 155.75 & $=00=$ & $0===$ & $===0$ & $01=0$ & $=110$ & $01==$ & $==00$ & \\
\hline
\end{tabular}

\footnotetext{
${ }^{2}$ The shortest TCEC-draw was TeXEL-Gull in Season 10, Stage 1. After the mandated 1. b4 d5 2. Bb2 Qd6, play went 3. a3 a5 4. Nf3 axb4 5. Be5 Qb6 6. Bd4 Qd6 7. Be5 Qb6 8. Bd4 Qd6 9. Be5 \{3x $\}$ 1/2-1/2.

${ }^{3}$ g45/12.1, AS-Ar: 1. e4 e5 2. Nf3 Nc6 \{as mandated 3. Nc3 Nf6 4. d4 exd4 5. Nxd4 Bb4 6. Nxc6 bxc6 7. Qd4? (7. Bd3) Qe7 8. f3 Bc5 9. Qd3 O-O 10. Bg5 Rb8 11. O-O-O d5 12. exd5 h6 13. Bh4 Ba6 14. Bxf6 Be3+ 15. Kb1?? (15. Rd2) Rxb2+ 16. Ka1 gxf6 17. Qxa6 Rfb8 18. Bd3?? (18. Qa5) Qb4 19. Ne2 Rxa2+ 20. Kxa2 Qb2\# 0-1.

${ }^{4}$ GULL's role is similar to that of the 'IPK' International Prototype Kilogram which defined the mass of one kilogram. If it hypothetically lost a gram, everything else would increase in mass by $0.1 \%$ even though unchanged. This parallel ceased to be valid on $20^{\text {th }}$ May 2019 when the new definition of the Kilogram became operative (BIPM, 2019).
} 


\section{Division 2: 2 DRR phases, 14 rounds, 112 games @ 30'+5"/m, 8-ply openings}

A burnished ALLIESTEIN checked in for Division 2 with endgame table support and without the castlingrelated bug that manifested itself in Division 3: ROFCHADE was also refreshed. A point of interest was whether the newly promoted engines would hang on to their new status.

After RR1, ALLIESTEIN headed the standings with XIPHOS on +3 despite losing the drawn g21/5.1 to ROFCHADE after 109. Ke5?? instead of Ke7. ROFCHADE itself was on -2 and in the drop zone. At the halfway point, the leaders were XipHOS (+7), AllieStein (+5) and CheSsBrainVB (+2), despite the fact that ALLIESTEIN lost to ROFCHADE again. Can an engine play so badly that it plays well?

In the third round robin, there were few upsets. CHESSBRAINVB lost to FRITZ and ceded third place to BoOOT $(+2)$ which had previously had difficulty winning. ALLIESTEIN $(+8)$ and XIPHOS $(+11)$ took their promotion prospects past the $97.6 \%$ and $99.9 \%$ confidence-levels respectively. NIRVANA was similarly headed for Division 3. In RR4, ROFCHADE escaped the drop in the final cliffhanger at the expense of FRITZ. XIPHOS and ALLIESTEIN gained promotion easily as BOOOT fell away badly. Interestingly, ALLIESTEIN performed better than XIPHOS against the better engines. Would this trend continue in Division 1 ?

Table 6. The TCEC15 Division 2 cross-table: two DRR phases, 28 rounds, 112 games.

\begin{tabular}{|c|c|c|c|c|c|c|c|c|c|c|c|c|c|c|}
\hline \# & Engine & ELO & Pts & P\% & Elo \pm & SB & $\mathbf{X i}$ & AS & Gi & Bo & $\mathbf{C b}$ & ro & $\mathbf{F r}$ & $\mathbf{N i}$ \\
\hline 1 & Xiphos 0.5.2 & 3449 & 19.5 & 69.6 & +21 & 240.25 & & $=0=0$ & $=1==$ & $==10$ & $1=11$ & $111=$ & $=111$ & $111=$ \\
\hline 2 & AllieStein v0.2-n4 & 3329 & 19.0 & 67.9 & +69 & 255.75 & $=1=1$ & & $11=1$ & $1===$ & $1=1=$ & $001=$ & $==1=$ & $1=1=$ \\
\hline 5 & Ginkgo 2.18 & 3440 & 15.0 & 53.6 & -20 & 181.75 & $=0==$ & $00=0$ & & $==01$ & $====$ & $1=1=$ & $0==1$ & 1111 \\
\hline 3 & Booot 6.3 .1 & 3407 & 14.0 & 50.0 & -16 & 187.50 & $==01$ & $0===$ & $==10$ & & $====$ & $===0$ & $=1=0$ & $1=1=$ \\
\hline 4 & ChessBrainVB 3.72 & 3342 & 14.0 & 50.0 & +13 & 172.50 & $0=00$ & $0=0=$ & $====$ & $====$ & & $11==$ & $=101$ & $1=1=$ \\
\hline 6 & rofChade $2.018 \mathrm{~T}$ & 3347 & 12.0 & 42.9 & -9 & 161.75 & $000=$ & $110=$ & $0=0=$ & $===1$ & $00==$ & & $====$ & $==1=$ \\
\hline 7 & Fritz 16.10 & 3319 & 11.5 & 41.1 & -1 & 155.25 & $=000$ & $==0=$ & $1==0$ & $=0=1$ & $=010$ & $====$ & & $====$ \\
\hline 8 & Nirvana 2.4 & 3343 & 7.0 & 25.0 & -57 & 97.75 & $000=$ & $0=0=$ & 0000 & $0=0=$ & $0=0=$ & $==0=$ & $====$ & \\
\hline
\end{tabular}

\section{Division 1: 2 DRR phases, 28 rounds, 112 games, tempo $60^{\prime}+5^{\prime \prime} / \mathrm{m}$}

Second author 'Cato' provided 12-ply openings for this division. The news was that the KOMODOMCTS crash problem was solved and so expectations were that it would promote easily. After seven rounds, KomodoMCTS led ALLIESTEIN and FIZBO, ALLIESTEIN second courtesy of a default by CHIRON in game 25 when the latter failed to play a single move and crashed after 2.5 minutes: hash-table initialisation may have been the problem. CHIRON has been on the cusp of Divisions 1 and P since it crashed three times in Season 12. KOMODOMCTS and FIZBO did not know that they were less likely to receive a similar gift but CHIRON was then cut back to 32 cores.

ALLIESTEIN (+4) reached half time strongly with $2.5 / 3$ and a key win against FIZBO. KOMODOMCTS $(+3)$ was second after being gifted a win by CHIRON in g46/12.2. With 12 moves to go to a 50-move draw, CHIRON lowered the drawbridge of its own fortress with 138. ... f6?? and welcomed its enemy in with red carpet and heralds. As Karsten Müller confirmed, Ke8 was always available to guarantee the draw. LASER (+1) was gapped in third, sound, unbeaten but draw-centric. JONNY and CHIRON looked earmarked for Division 2 with just one win, literally between them. 
Round Robin 3 ended with fireworks, nine of twelve games being decisive. ALLIESTEIN's first win of RR3, a key one in g77/20.1 against XIPHOS, was followed by a network crash against CHIRON at move 70 in $\mathrm{g} 81 / 21.1$. This was posted as a 'no fault' loss but continued later and was drawn as expected. Meanwhile, KOMODOMCTS had moved confidently back to first place with three straight wins, the last to previously undefeated LASER. XIPHOS and LASER contested third place but were effectively two points behind ALLIESTEIN because of tiebreaks.

The last quarter started badly for ALLIESTEIN: its first genuine loss in g85/22.1 against ANDSCACS (Wool, 2019), a sharp, tactical Q-RR fight, not its forte. However, its win against KOMODOMCTS in g93/24.1 more than revived its promotion chances, giving it tie-breaking advantage against the other contenders. ALLIESTEIN and XIPHOS met in g105/27.1 for almost all the marbles and drew. As the dust settled and the GPUs cooled, positions 1-5 were decided clearly on points despite the closeness of this division. FIZBO escaped relegation only by virtue of the third 'number of wins' tiebreaker at the expense of JONNY which had the better SB score: TCEC follow FIDE's tie-break priorities here. CHIRON, tailed off last at -10 and with one more albeit ultimately irrelevant crash to its name, now finds itself two divisions below its personal zenith. The two promotion spots went to KOMODOMCTS (+7) and newcomer ALLIESTEIN (+5) with fellow promotee XIPHOS (+3) a creditable third.

Table 7. The TCEC15 Division 1 cross-table: two DRR phases, 28 rounds, 112 games.

\begin{tabular}{|c|c|c|c|c|c|c|c|c|c|c|c|c|c|c|}
\hline If & ngine & ELO & Pts & $\mathbf{P \%}$ & Elo \pm & SB & $\mathbf{K m}$ & AS & $\mathbf{X i}$ & $\mathbf{L a}$ & An & $\mathbf{F z}$ & Jo & Ch \\
\hline 1 & omodoMCTS 2301.00 & 487 & 17.5 & 62.5 & +25 & 229.75 & & $===0$ & $10==$ & $==1=$ & $11==$ & $==11$ & $===1$ & $=11=$ \\
\hline 2 & & 00 & .5 & & +84 & 223 & $===1$ & & & & $==0$ & $=1==$ & 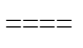 & $11==$ \\
\hline 3 & Kip & 467 & 5.5 & 55.4 & +3 & 204.75 & $01==$ & & & $====$ & $=1=$ & $01=$ & & $=11$ \\
\hline 4 & Lase & 3444 & 15.0 & 53.6 & +14 & 199.00 & $==0=$ & $====$ & $====$ & & $===0$ & $==1=$ & $=1==$ & $==11$ \\
\hline 5 & Ands & 469 & 14.5 & 51.8 & -19 & 194.50 & $00==$ & $===1$ & $==0=$ & $===1$ & & $01==$ & $==10$ & $11==$ \\
\hline 6 & Fizbo & 419 & 12.0 & 42.9 & -23 & 163.50 & $==00$ & $=0==$ & $010=$ & $==0=$ & $10==$ & & $1==0$ & $==1=$ \\
\hline 7 & Jonny 8.1 & 3400 & 12.0 & 42.9 & -6 & 171.50 & $===0$ & $====$ & $==0=$ & $=0==$ & $==01$ & $0==1$ & & $=0==$ \\
\hline 8 & Chiron 230119 & 3411 & 9.0 & 32.1 & -76 & 127.00 & $=00=$ & $00==$ & $==00$ & $==00$ & $00==$ & $==0=$ & $=1==$ & \\
\hline
\end{tabular}

\section{Division $P$, three DRR phases, 42 rounds, 168 games, tempo $90^{\prime}+5^{\prime \prime} / \mathrm{m}$}

The heavyweight Division P promised three weeks of the best computer chess to be found anywhere. It featured three non-Shannon (1950) MCTS engines: TCEC14 runner-up 'LC0' LEELA CHESS ZERO, returning KOMODOMCTS and serial promotee ALLIESTEIN. The majority of games, those between the tactical minimaxers and the strategic Monegasques, were bound to be a clash of two styles and particularly interesting. After three rounds, STOCKFISH, LC0 and HOUDINI shared the three wins: the stablemates KOMODO and KOMODOMCTS were also unbeaten. In g24/6.4, ALLIESTEIN posted the first win for Black, an apparent draw at m74 but a promising R-BPPP endgame at m80 closed out only after another 90 moves of suspenseful exploration. Round Robin 1 featured just five precious wins and left four engines on +1 with only ETHEREAL (-1) and FIRE (-2) in deficit. The three 'all-MCTS' games were each drawn in over 112 moves.

After RR2 which sported seven wins, STOCKFISH (+4) and LC0 (+3) opened up on KoMODOMCTS and HOUDINI (+1). KOMODO, a three-time TCEC Champion redefined 'solid' on 14 draws from 14, only good enough for $6^{\text {th. }}$ : ETHEREAL (-3) and FIRE (-5) were the principal donors and looked like joint tenants of the basement. 'MCTS v Shannon' with 9 of the 12 decisive games stood at $+6=21-3$. LC 0 beat ALLIESTEIN, g39/10.3. All the red ink was below the x-table diagonal. 
In round robin 3, STOCKFISH scored wins over the previously unbeaten KOMODO and HOUDINI, and over ETHEREAL and FIRE - a complete take-out of the last four. All others were \pm 1 except ETHEREAL which lost touch with -3. ALLIESTEIN dropped a win against KOMODOMCTS in g19.2 with 36. Re1? instead of 36. Kh4! Both engines were against the clock in the drawn FIRE-ALLIESTEIN g80/20.4 until 189. ... Kf5?? was preferred to the essential Kh6. This was the longest TCEC15 win to date and the first win by the underdog.

The fourth round robin resulted in a clear 2-4-2 formation: STOCKFISH and LEELA well out front, FIRE and ETHEREAL detached, and the remaining four on -1. We saw the shortest sequence of played moves in TCEC15: after the provided opening of g107/27.3, ETHEREAL demolished KOMODOMCTS in an amazing 24 moves. The latter never seemed to be on terms with the situation. The MCTS-Shannon match moved on to $+9=45-6$ with three wins each in RR3-4.

With the Superfinal and demotions essentially if not formally decided, interest in the last third of Division P perhaps focused on the midfield contest. KOMODO rose while KOMODOMCTS fell, crashing against ALLIESTEIN and losing quickly again, this time to STOCKFISH. LEELA beat STOCKFISH to win their head-to-head: what did this say about the Superfinal to come?!

This is an appropriate moment to recommend Assaf Wool's (2019) coverage of the TCEC games. For this Premier Division, Wool touched on all decisive games and some draws. He particularly focused on game 6 (HoudinI-ALLIESTEIN, 1-0), g37 (STOCKFISH-KOMODOMCTS, 1-0), another clash of styles, g57 (KOMODO-STOCKFISH, 0-1), g86 (LEELA-KOMODOMCTS, 1-0), g127 (KOMODO-ALliESTEIN, 1-0) and g161 (LEELA-STOCKFISH, 1-0).

And so the stage is set for a repeat of the TCEC14 Superfinal. LEELA comes through unbeaten with a positive score against all except HOUDINI. This is a remarkable achievement in a heavyweight division: the average game length of some 80 moves and median of 70 moves indicates that games were on the whole closely contested.

Table 8. The TCEC15 Premier Division cross-table: three DRR phases, 42 rounds, 168 games.

\begin{tabular}{|c|c|c|c|c|c|c|c|c|c|c|c|c|c|}
\hline \# & Engine & ELO & Pts & $\mathrm{P} \%$ & Elo \pm & $\mathbf{S t}$ & Lc & Ko & AS & Ho & Km & Et & Fi \\
\hline 1 & Stockfish 19040612 & 3603 & 27.5 & 65.5 & 49 & & $=====0$ & $==11==$ & $=1=1==$ & $==111=$ & $=1===1$ & $1=1===$ & $=11==1$ \\
\hline 2 & LCZero v0.21.1-n41800 & 3603 & 25.5 & 60.7 & -15 & $====1$ & & $====1=$ & $=1===1$ & $======$ & $===1==$ & $=11===$ & $1===1=$ \\
\hline 3 & Komodo 2306.00 & 3561 & 21.5 & 51.2 & -55 & $==00==$ & $====0=$ & & $====1=$ & $=====$ & $======$ & $===1==$ & $====11$ \\
\hline 4 & AllieStein v0.3dev-n6.1 & 3426 & 20.5 & 48.8 & 197 & $=0=0==$ & $=0===0$ & $====0=$ & & $0==1==$ & $====1=$ & $==1=1=$ & $110===$ \\
\hline 5 & Houdini 6.03 & 3571 & 20.5 & 48.8 & -108 & $==000=$ & $=====$ & $=====$ & $1==0==$ & & $=====$ & $===1==$ & $=====1$ \\
\hline 6 & KomodoMCTS 2306.00 & 3486 & 19.5 & 46.4 & 41 & $=0==-0$ & $===0==$ & $=====$ & $====0=$ & $======$ & & $=1=0==$ & $1====$ \\
\hline 7 & Ethereal 11.38 & 3483 & 17.0 & 40.5 & -33 & $0=0===$ & $=00===$ & $==0==$ & $==0=0=$ & $==0==$ & $=0=1==$ & & $===01=$ \\
\hline 8 & Fire 011819 & 3488 & 16.0 & 38.1 & -75 & $=00==0$ & $0===0=$ & $====00$ & $001===$ & $=====0$ & $0=====$ & $===10=$ & \\
\hline
\end{tabular}

Table 9. The TCEC15 Premier Division figures: head-to-head and round-by-round scores.

\begin{tabular}{|c|c|c|c|c|c|c|c|c|c|c|c|c|c|c|c|c|c|c|c|}
\hline \# & Engine & ELO & Pts & SB & St & Lc & Ko & AS & Ho & Km & Et & $\mathbf{F i}$ & RR... & $\mathbf{r 1}$ & r2 & r3 & r4 & r5 & r6 \\
\hline 1 & Stockfish 19040612 & 3603 & 27.5 & 542.00 & & $21 / 2$ & 4 & 4 & $41 / 2$ & 4 & 4 & $41 / 2$ & & 4 & 5 & $51 / 2$ & 5 & 4 & 4 \\
\hline 2 & LCZero v0.21.1-n41800 & 3603 & 25.5 & 515.25 & $31 / 2$ & & $31 / 2$ & 4 & 3 & $31 / 2$ & 4 & 4 & & 4 & $41 / 2$ & 4 & 4 & $41 / 2$ & $41 / 2$ \\
\hline 3 & Komodo 2306.00 & 3561 & 21.5 & 434.00 & 2 & $21 / 2$ & & $31 / 2$ & 3 & 3 & $31 / 2$ & 4 & & $31 / 2$ & $31 / 2$ & 3 & $31 / 2$ & 4 & 4 \\
\hline 4 & AllieStein v0.3dev-n6.1 & 3426 & 20.5 & 413.50 & 2 & 2 & $21 / 2$ & & 3 & $31 / 2$ & 4 & $31 / 2$ & & $31 / 2$ & 3 & $31 / 2$ & $31 / 2$ & 4 & 3 \\
\hline 5 & Houdini 6.03 & 3571 & 20.5 & 417.75 & $1 \frac{1}{2}$ & 3 & 3 & 3 & & 3 & $31 / 2$ & $31 / 2$ & & 4 & $31 / 2$ & 3 & 3 & 3 & 4 \\
\hline 6 & KomodoMCTS 2306.00 & 3486 & 19.5 & 403.00 & 2 & $21 / 2$ & 3 & $2 \frac{1}{2}$ & 3 & & 3 & $31 / 2$ & & 4 & $31 / 2$ & $31 / 2$ & $21 / 2$ & 3 & 3 \\
\hline 7 & Ethereal 11.38 & 3483 & 17.0 & 358.50 & 2 & 2 & $21 / 2$ & 2 & $2^{1 / 2}$ & 3 & & 3 & & 3 & $21 / 2$ & 2 & $21 / 2$ & $31 / 2$ & $31 / 2$ \\
\hline 8 & Fire 011819 & 3488 & 16.0 & 337.50 & $1 \frac{1 / 2}{2}$ & 2 & 2 & $21 / 2$ & $21 / 2$ & $21 / 2$ & 3 & & & 2 & $2 \frac{1}{2}$ & $31 / 2$ & 4 & 2 & 2 \\
\hline
\end{tabular}


Again, after an intermission for the knockout TCEC Cup 3 (Haworth and Hernandez, 2019a), both STOCKFISH and LEELA CHESS ZERO came to the Superfinal in new versions. LEELA had won the last two TCEC Cup events, and a 'bonus, no opening book' match against STOCKFISH at the same Rapid tempo. Probably because of the very close TCEC14 result, a win by the smallest possible margin of one game, and the fact that LEELA was expected to have improved more than STOCKFISH, the six-time TCEC champion was no longer the favourite in the initial straw-poll.

Some evidence that LEELA was stronger in the endgame came to hand before the Superfinal started. The pause after TCEC Cup 3 included a replaying of the TCEC14 Superfinal game 65 from the 7-man KNPPKBP position after 73. Kxf3. In the actual Superfinal, LEELA did not latch on to the key winning ideas quickly enough even with the help of the 6-man EGTs: the 50-move rule intervened. In 'bonus mode', the endgame was revisited with a later version of LEELA and this time, LEELA secured the win with less than half its previous inaccuracies. Fig. 2 shows the tracks of the two contests, depth in plies plotted against plies played. Optimal play is also shown for comparison.

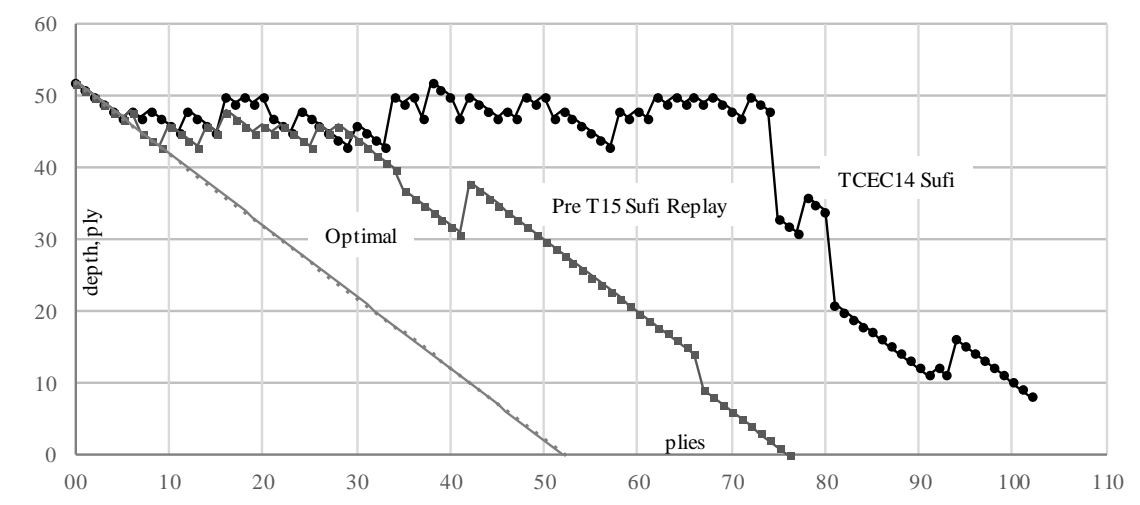

Fig. 2. TCEC14 game 65 from KNPPKBP position 73b: (a) as played in the Sufi, (b) as replayed and (c) optimal play.

This Superfinal was even for the first 15 games with one win to each side. STOCKFISH ominously opened its account with a win as Black. The expected close contest was in prospect but games 16-26 saw four wins by LEELA without reply. With hindsight, this was where most of the damage was done. Games 3545 saw a flurry of decisive games with STOCKFISH pulling one back overall to improve the mood in its fan base. However, LEELA struck with back-to-back wins in games 61-62: shall we hear why the Trompowsky Attack, also associated with Ruth and Opočenský, seems not to suit STOCKFISH?

Thoughts of a comeback were rather dulled by eighteen draws but then STOCKFISH won again and in spectacular fashion with game 81. After 27. ... Rae8, STOCKFISH saw a clear win with 26. h6 which LEELA had equally clearly missed. Sure enough, LEELA had to lose queen for knight in order to create the merest distraction. This was not the first time the new-style ANN engines had failed to find a sharp, tactical needle during a Monte-Carlo Tree-Search. The match was clearly not over. If LEELA could score four in short order, so could STOCKFISH. In fact, this did not happen. LEELA hit back immediately in game 82 and added insult to injury with two more wins in games 88 and 94.

'The king is dead: long live the Queen'. Table 10 and Fig. 3 provide the core data. The final score of $53 \frac{1}{2}-46 \frac{1}{2}$ was more decisive than expected and it is easy to think that STOCKFISH did not play well. This of course is not the case: LEELA just played better, some 27 ELO ahead in Implied Performance 
terms. Table 11 shows that games were a superhuman 89 moves long on average, not the 99 moves of their TCEC14 contest but the incremental time was less and the games were 20' shorter. Energy drinks please, not for the players but for the audience. Clearly, TCEC Superfinals are increasingly attracting top players to TCEC and we will hear more of these games. 'Kingscrusher' (2019b-2019u), known to his parents and now to others as CM Tryfon Gavriel, continues to provide richly informative videocommentaries. The 'Kingscrusher' (2019a) interview with Game Changer's Matthew Sadler and Natasha Regan (2019) is also relevant to LEELA's play. Wool (2019) and 'GM TheChessPuzzler' (2019) are also making extended and valuable contributions. Long may these continue.

Demis Hassabis' (2019) tweet "Great to see learning systems come out on top. Huge congrats to the @LeelaChessZero team and community!” was typically enthusiastic and encouraging.

Table 10. The TCEC 15 Superfinal match of 100 games: the decisive games, Black wins underlined.

\begin{tabular}{|c|c|c|c|c|c|c|}
\hline Superfinal & ELO Score & Perf. ELO $\Delta$ & \# of games won (underline = ' $0-1$ ') & Openings & \# of game-pairs won & \# of win-pairs \\
\hline $\begin{array}{l}\text { Leela Chess Zero } \\
0.21 .1-\mathrm{nT} 40 . \mathrm{T} 8.610\end{array}$ & $53 \frac{112}{2}$ & $53.5 \%$ & $\begin{array}{l}10,16,18,24,26,36,38,40 \\
\underline{61}, 62,82,86,88,94\end{array}$ & $\begin{array}{l}\text { A67, B06, E97, C92, E15, B90, } \\
\text { A21, C19, A45, A45, E88, A89, }\end{array}$ & $9 \begin{array}{l}5,8,9,12,13 \\
19,31,43,47\end{array}$ & $\begin{array}{l}35-36,39- \\
40-61-62\end{array}$ \\
\hline Stockfish 19050918 & 3587 & $46.5 \%^{*}-14$ & $7 \underline{12}, 35,39,43,45,81,87$ & $\begin{array}{l}\text { C05, B90, C19, C57, A50, E87, } \\
\text { A42 }\end{array}$ & $36,22,23$ & $\begin{array}{c}40, \underline{61-62,} \\
81-82,87-88\end{array}$ \\
\hline
\end{tabular}

Fig. 3. The TCEC15 Superfinal: the incidence of decisive results and LEELA CHESS ZERO's lead.
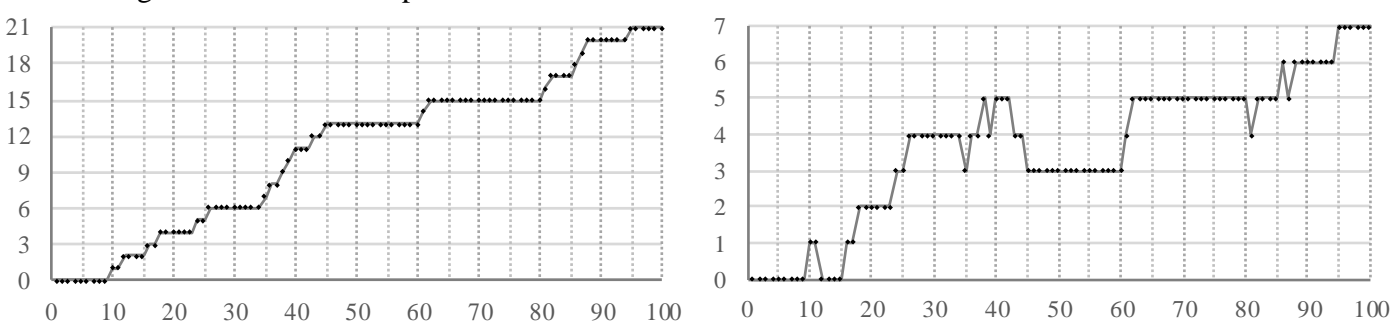

Table 11. Generic statistics for each phase of TCEC15: results, terminations and average game-length.

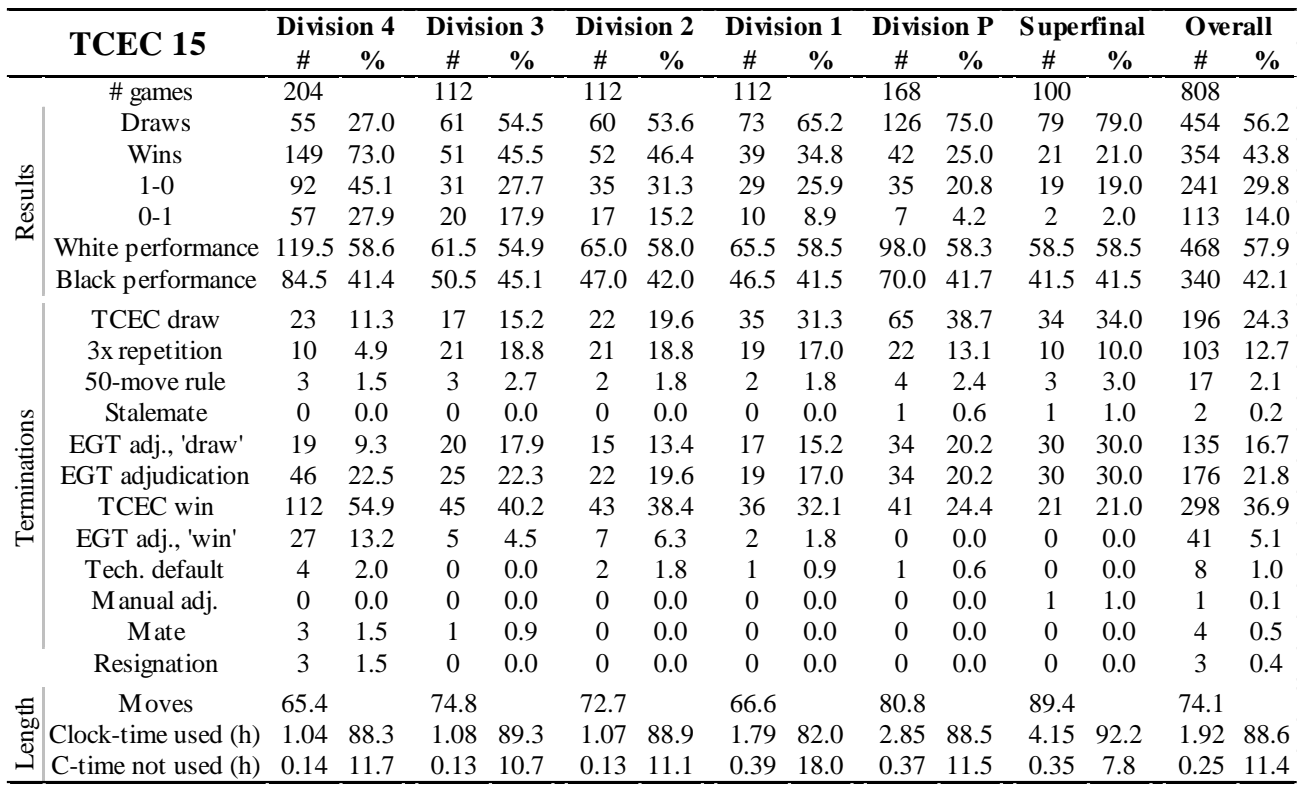


After fifteen seasons of TCEC, it is worth reflecting that league tables tend to emphasise the ranking of chess engines rather than their relative differences, and certainly rather than their absolute prowess. Also, given the nearly non-stop nature of TCEC events, it is easy to forget that hours of top-level chess are passing before us in all the divisions - and inevitably, without getting the attention and analysis that they deserve, despite the hints on the TCEC and Chessbomb (2019) sites and Sadler's (2019) perspective. Any of the TCEC15 engines would give a Grandmaster a serious game and most would perhaps have to be handicapped by a Blitz or even Armageddon tempo. Even so, there is evidence here that if there is some 'ceiling' asymptote to quality of play, it is still some way off. The admirable Emil Vlasák (2019) has, for example, clearly demonstrated that LEELA would not be at all competitive in a Computer-solving Championship as it struggles to find study-like wins.

Congratulations to TCEC's new champion, LEELA CHESS ZERO, and to all who have assisted in her conception and evolution. STOCKFISH, champion for TCEC seasons 6, 9 and 11-14, continues to dominate the rest of the field and we can expect to see hostilities renewed. Perhaps LEELA's vulnerability to tactical shots will be exposed further. 'Kudos' to all other participants and to the core TCEC team who make all this happen.

Will further engines of the 'new genre' join the fray, and will hybrid engines appear, incorporating the best of the 'minimax' and MCTS perspectives? Will TCEC be able to combine the strengths of their two servers in one platform in order to facilitate this? Will computer-based tools emerge to help us understand the subtleties of the games we are witnessing? We shall see. All TCEC15 games, many with additional annotation and play-outs, are available in pgn form together with data beyond that of Tables 11-12 (Haworth and Hernandez, 2019b).

Table 12. The shortest and longest 1-0, drawn and 0-1 games in each phase of TCEC15. Game indication: 'c7/4.1' for example means Division 4c, pgn game 7, round 4, game 1.

\begin{tabular}{|c|c|c|c|c|c|c|c|c|c|c|c|c|c|c|c|c|c|c|}
\hline & \multicolumn{6}{|c|}{ 1-0 } & \multicolumn{6}{|c|}{$1 / 2-1 / 2$} & \multicolumn{6}{|c|}{$0-1$} \\
\hline & \multicolumn{3}{|c|}{ Shortest } & \multicolumn{3}{|c|}{ Longest } & \multicolumn{3}{|c|}{ Shortest } & \multicolumn{3}{|c|}{ Longest } & \multicolumn{3}{|c|}{ Shortest } & \multicolumn{3}{|c|}{ Longest } \\
\hline & $\mathbf{G a}$ & & $\# \mathbf{m v}$ & $\mathbf{G a}$ & me & $\# \mathbf{m v}$ & Ga & me & \#mv & Ga & & $\# \mathbf{m v}$ & Gar & & $\# \mathbf{m v}$ & Gar & & $\# \mathbf{m v}$ \\
\hline 4 & c7/4.1 & AS-Ne & 29 & a54/11.4 & To-Mi & 135 & b41/9.1 & AS-Ga & 38 & b46/10.1 & Ga-pi & 135 & b13.5/65 & To-Tu & 31 & b12.5/60 & Mo-Sn & 188 \\
\hline 3 & $59 / 15.3$ & Ar- & 35 & & & 172 & $35 / 4$ & & 16 & & & 185 & & & 20 & & & 198 \\
\hline 2 & $92 / 23.4$ & Gi-Fr & 45 & $80 / 20.4$ & $\mathrm{Xi}-\mathrm{Bo}$ & 180 & $53 / 14.1$ & AS & 23 & $85 /$ & AS-Fr & 244 & $99 / 25.3$ & Bo-Fr & 46 & & & 134 \\
\hline 1 & $84 / 21.4$ & Xi-An & 38 & $46 / 12.2$ & $\mathrm{Km}-\mathrm{Ch}$ & 153 & $48 / 12.4$ & Xi-Jo & 15 & $13 / 4.1$ & AS-Jo & 180 & $100 / 25.4$ & Fi-Km & 39 & $60 / 15.4$ & Ch-La & 83 \\
\hline $\mathbf{P}$ & $107 / 27.3$ & Et-Km & 32 & $80 / 20.4$ & Fi-AS & 214 & $167 / 42.3$ & $\mathrm{Km}-\mathrm{Ho}$ & 25 & $112 / 28.4$ & Ko-Lc0 & 217 & $51 / 13.3$ & Et-Km & 68 & $24 / 6.4$ & Fi-AS & 170 \\
\hline SF & $81 / 81$ & St-Lc & 41 & $16 / 16$ & Lc-St & 143 & $67 / 67$ & St-Lc & 38 & $33 / 33$ & St-Lc & 262 & $12 / 12$ & Lc-St & 81 & $61 / 61$ & St-Lc & 82 \\
\hline O'all & $4 c, 4.1$ & AS-Ne & 29 & $\mathrm{P}, 20.4$ & $\mathrm{Fi}-\mathrm{AS}$ & 214 & $2,12.4$ & $\mathrm{Xi-Jo}$ & 15 & $2,22.1$ & AS-Fr & 244 & $3,12.1$ & AS-Ar & 20 & $3,23.2$ & $\mathrm{Ne}-\mathrm{Te}$ & 198 \\
\hline
\end{tabular}

\section{REFERENCES}

BIPM (2019). https://preview.tinyurl.com/y3thmdyk. 'New SI' implementation day, 2019-05-20.

CPW (2019). https://tinyurl.com/icga046. The Chess Programming Wiki website, including biographies of engines, authors and developers.

Chessbomb (2019). https://www.chessbomb.com/arena/-/2019-tcec-s15. TCEC15 coverage.

Chessdom (2019). https://tinyurl.com/icga058. Chessdom interview with the ALLIESTEIN team.

de Man, R. (2018). http://tablebase.sesse.net/syzygy/. Site providing sub-8-man DTZ 50 " EGTs.

'GM Thechesspuzzler' (2019). https://tinyurl.com/tcec-gcp001. TCEC15 Superfinal commentaries.

Hassabis, D. (2019). https://tinyurl.com/tcec-dm001. DH's twittered congratulations to team LEELA. 
Haworth, G. $M^{\mathrm{c} C}$. and Hernandez, N. (2019a). http://centaur.reading.ac.uk/83157/. TCEC Cup 3. The third TCEC knockout Cup. ICGA Journal, 41(3), 168-173. doi:10.3233/ICG-190114.

Haworth, G. $M^{\mathrm{c} C}$. and Hernandez, N. (2019b). http://centaur.reading.ac.uk/83156/. This note plus annotated statistics and pgn files. ICGA Journal, 41(3), 153-163. doi:10.3233/ICG-190115. Intel (2017). https://tinyurl.com/icga042. Intel's specification of the XEON ${ }^{\circledR}$ E5-2699V4 processor.

'Kingscrusher' (2019a). https://tinyurl.com/tcec-kc003. Interview about the book Game Changer.

'Kingscrusher' (2019b). https://tinyurl.com/tcec-kc004. TCEC15 Superfinal videos.

'Kingscrusher' (2019c). https://tinyurl.com/tcec-kc005. Sufi g08: notable, fighting draw.

'Kingscrusher' (2019d). https://tinyurl.com/tcec-kc006. Sufi g10: Benoni novelty 12. Be2.

'Kingscrusher' (2019e). https://tinyurl.com/tcec-kc007. Sufi g12: LC0 blunder with 67.Kf3.

'Kingscrusher' (2019f). https://tinyurl.com/tcec-kc008. Sufi g16: LC0 accumulates advantages.

'Kingscrusher' (2019g). https://tinyurl.com/tcec-kc009. Sufi g18: LC0 Bayonet attack, m40.

'Kingscrusher' (2019h). https://tinyurl.com/tcec-kc010. Sufi g24: LC0 channels Karpov to win.

'Kingscrusher' (2019i). https://tinyurl.com/tcec-kc011. Sufi g26: LC0 wins RB-NN endgame.

'Kingscrusher' (2019j). https://tinyurl.com/tcec-kc012. Sufi g36: 'best game so far' epithet.

'Kingscrusher' (2019k). https://tinyurl.com/tcec-kc013. Sufi g38: ST loses with doubled pawns.

'Kingscrusher' (20191). https://tinyurl.com/tcec-kc014. Sufi g39: tactically-rich STOCKFISH win.

'Kingscrusher' (2019m). https://tinyurl.com/tcec-kc015. Sufi g40: LC0's positional play wins.

'Kingscrusher' (2019n). https://tinyurl.com/tcec-kc016. Sufi g43: LC0 resistance collapses $\sim \mathrm{m} 78$.

'Kingscrusher' (2019o). https://tinyurl.com/tcec-kc017. Sufi g45: ST seals win with 87. f4. 8-5.

'Kingscrusher' (2019p). https://tinyurl.com/tcec-kc018. Sufi g61: LC0's three-passed-pawn win.

'Kingscrusher' (2019q). https://tinyurl.com/tcec-kc019. Sufi g62: ‘double’ win, more passers.

'Kingscrusher' (2019r). https://tinyurl.com/tcec-kc020. Sufi g81: LC0 loses Queen to tactics.

'Kingscrusher' (2019s). https://tinyurl.com/tcec-kc021. Sufi g82: LC0 sees advantage, m19

'Kingscrusher' (2019t). https://tinyurl.com/tcec-kc022. Sufi g86: LC0 wins, outside passer.

'Kingscrusher' (2019u). https://tinyurl.com/tcec-kc023. Sufi g87: STOCKFISH's last win.

Nvidia (2018). https://www.nvidia.com/en-gb/geforce/graphics-cards/rtx-2080-ti/ GEFORCE GTX 2080 TI GPU specification and benchmark performance data.

Sadler, M. (2019). The TCEC15 Computer Chess Superfinal: a perspective. ICGA Journal, 41(3), 164-167. doi:10.3233/ICG-190112.

Sadler, M. and Regan, N. (2019). Game Changer: AlphaZero's Groundbreaking Chess Strategies and the Promise of AI. New in Chess. ISBN 978-90-5691-818-7.

Shannon, C. E. (1950). Programming a Computer for Playing Chess. The London, Edinburgh and Dublin Philosophical Magazine, 41(314), 256-275. https://doi.org/10.1080/14786445008521796.

Vlasák, E. (2019). LEELA CHESS ZERO. EG, Vol. 25(216), 106-8.

Wool, A. (2019) http://mytcecexperience.blogspot.co.uk/. AW’s ‘TCEC Experience’ blog. 